\title{
A Procedure for Obtaining a Family of Iterative Formulas for Finding Zeros of Functions
}

\author{
Dragomir M. Simeunović
}

\begin{abstract}
In this paper a family of iterative formulas for finding zeros of functions is obtained. The family includes the Laguerre method. All the methods of the family are cubically convergent for a simple zero. The superior behavior of Laguerre method when starting from the point $x_{k}$ for which $\left|x_{k}\right|$ is large, is also explained.
\end{abstract}

In this paper for finding the real simple zero of the function $f(x)$ we use the function

$$
F(x)=\frac{f(x)}{(x+c)^{s}},
$$

where $c$ and $s(s \neq 0)$ are real parameters, because the zero of $F(x)$ is also the zero of $f(x)$.

For finding a real simple zero $r$ of the function $F(x)$ we apply the Newton method (method of the tangents)

$$
x_{k+1}=x_{k}-\frac{F\left(x_{k}\right)}{F^{\prime}\left(x_{k}\right)}, \quad k=0,1,2, \ldots,
$$

with the condition

$$
F^{\prime \prime}\left(x_{k}\right)=0,
$$

where $x_{k}$ is the approximative value of the zero $r$ of the function $F(x)$, that is, the zero $r$ of the function $f(x)$.

From (1) we obtain

$$
\begin{aligned}
F^{\prime}(x) & =\frac{(x+c) f^{\prime}(x)-s f(x)}{(x+c)^{s+1}} \\
F^{\prime \prime}(x) & =\frac{(x+c)^{2} f^{\prime \prime}(x)-2 s(x+c) f^{\prime}(x)+s(s+1) f(x)}{(x+c)^{s+2}} .
\end{aligned}
$$

The condition (3) reduces to equation

$$
\left(x_{k}+c\right)^{2} f^{\prime \prime}\left(x_{k}\right)-2 s\left(x_{k}+c\right) f^{\prime}\left(x_{k}\right)+s(s+1) f\left(x_{k}\right)=0,
$$

2000 Mathematics Subject Classification. Primary: 65H05.

Key words and phrases. Iterative formulas, approximative solutions of equations. 
where $x_{k}$ is the approximative value of the zero $r$ of the function $f(x)$.

From equation (6) on obtain

$$
x_{k}+c=\frac{s f^{\prime}\left(x_{k}\right) \pm \sqrt{s^{2}\left(f^{\prime}\left(x_{k}\right)\right)^{2}-s(s+1) f\left(x_{k}\right) f^{\prime \prime}\left(x_{k}\right)}}{f^{\prime \prime}\left(x_{k}\right)},
$$

that is

$$
x_{k}+c=\frac{s(s+1) f\left(x_{k}\right)}{s f^{\prime}\left(x_{k}\right) \mp \sqrt{s^{2}\left(f^{\prime}\left(x_{k}\right)\right)^{2}-s(s+1) f\left(x_{k}\right) f^{\prime \prime}\left(x_{k}\right)}} .
$$

From (1) and (4) we obtain

$$
\frac{F\left(x_{k}\right)}{F^{\prime}\left(x_{k}\right)}=\frac{f\left(x_{k}\right)}{f^{\prime}\left(x_{k}\right)-\frac{s f\left(x_{k}\right)}{x_{k}+c}} .
$$

If $x_{k}+c$ from (8) we put in (9) we obtain

$$
\frac{F\left(x_{k}\right)}{F^{\prime}\left(x_{k}\right)}=\frac{(s+1) f\left(x_{k}\right)}{f^{\prime}\left(x_{k}\right) \pm \sqrt{s^{2}\left(f^{\prime}\left(x_{k}\right)\right)^{2}-s(s+1) f\left(x_{k}\right) f^{\prime \prime}\left(x_{k}\right)}} .
$$

In this case the formula (2) reduces to

$$
\begin{gathered}
x_{k+1}=x_{k}-\frac{(s+1) f\left(x_{k}\right)}{f^{\prime}\left(x_{k}\right) \pm \sqrt{s^{2}\left(f^{\prime}\left(x_{k}\right)\right)^{2}-s(s+1) f\left(x_{k}\right) f^{\prime \prime}\left(x_{k}\right)}}, \\
k=0,1,2, \ldots
\end{gathered}
$$

The formula (10) represents one family of iterative formulas for finding a real simple zero of the function $f(x)$.

All the methods of the family (10) are cubically convergent for a simple zero of the function $f(x)$.

The asymptotic error constant for family (10) is

$$
C_{3}=\frac{3\left(1-\frac{1}{s}\right)\left(f^{\prime \prime}(r)\right)^{2}-4 f^{\prime}(r) f^{\prime \prime \prime}(r)}{24\left(f^{\prime}(r)\right)^{2}} .
$$

For $s=\frac{1}{\alpha}$ from (10) on obtain the family

$$
\begin{gathered}
x_{k+1}=x_{k}-\frac{(\alpha+1) f\left(x_{k}\right)}{\alpha f^{\prime}\left(x_{k}\right) \pm \sqrt{\left(f^{\prime}\left(x_{k}\right)\right)^{2}-(\alpha+1) f\left(x_{k}\right) f^{\prime \prime}\left(x_{k}\right)}}, \\
k=0,1,2, \ldots
\end{gathered}
$$

The family (12) was derived by Eldon Hansen and Merrell Patrick in [2].

Now we consider the real polynomial

$$
P(x)=x^{n}+a_{1} x^{n-1}+a_{2} x^{n-2}+\cdots+a_{n-1} x+a_{n}
$$

whose zeros are all real and different. 
For $s=n-1$ and $f(x)=P(x)$, the function (1) reduces to

$$
F(x)=\frac{P(x)}{(x+c)^{n-1}} .
$$

In this case the iterative formula (10) reduces to the Laguerre formula [1], for $k=0,1,2, \ldots$

$$
x_{k+1}=x_{k}-\frac{n P\left(x_{k}\right)}{P^{\prime}\left(x_{k}\right) \pm \sqrt{(n-1)^{2}\left(P^{\prime}\left(x_{k}\right)\right)^{2}-n(n-1) P\left(x_{k}\right) P^{\prime \prime}\left(x_{k}\right)}},
$$

We can write (15) in the form

$$
\begin{gathered}
x_{k+1}=x_{k}-\frac{P\left(x_{k}\right)}{P^{\prime}\left(x_{k}\right)} \cdot \frac{n}{1 \pm(n-1) \sqrt{1-\frac{n}{n-1} \cdot \frac{P\left(x_{k}\right) P^{\prime \prime}\left(x_{k}\right)}{\left(P^{\prime}\left(x_{k}\right)\right)^{2}}}}, \\
\quad k=0,1,2, \ldots
\end{gathered}
$$

As we can see, the Laguerre formula (15), respectively formula (16) represent the Newton method (the method of tangents) applied to function (14), with the condition (3), respectively condition (6), which now reduces to condition

$$
\left(x_{k}+c\right)^{2} P^{\prime \prime}\left(x_{k}\right)-2(n-1)\left(x_{k}+c\right) P^{\prime}\left(x_{k}\right)+n(n-1) P\left(x_{k}\right)=0,
$$

where $x_{k}$ is the approximative value of the zero $r$ of the polynomial $P(x)$.

For $s=n-1$ and $f(x)=P(x)$ from (7) we obtain

$$
c=\frac{(n-1) P^{\prime}\left(x_{k}\right)-x_{k} P^{\prime \prime}\left(x_{k}\right) \pm \sqrt{(n-1)^{2}\left(P^{\prime}\left(x_{k}\right)\right)^{2}-n(n-1) P\left(x_{k}\right) P^{\prime \prime}\left(x_{k}\right)}}{P^{\prime \prime}\left(x_{k}\right)}
$$

If we put in (18) the corresponding values for $P\left(x_{k}\right), P^{\prime}\left(x_{k}\right)$ and $P^{\prime \prime}\left(x_{k}\right)$ obtained from (13), then for large $\left|x_{k}\right|$ we have

$$
c=\frac{a_{1}}{n} \pm \sqrt{\left(\frac{a_{1}}{n}\right)^{2}-\frac{2 a_{2}}{n(n-1)}}+O\left(\frac{1}{x_{k}}\right) .
$$

For $\left|x_{k}\right|=\infty$ from (19) for $c$ we obtain

$$
c_{a}=\frac{a_{1}}{n} \pm \sqrt{\left(\frac{a_{1}}{n}\right)^{2}-\frac{2 a_{2}}{n(n-1)}} .
$$

The tangent of the curve (14) at the point $\left|x_{k}\right|=\infty$ is its asymptote whose equation is

$$
y=x+a_{1}-(n-1) c_{a},
$$

because $c=c_{a}$ for $\left|x_{k}\right|=\infty$. 
According to (20) the equation (21) reduces to

$$
y=x+\frac{a_{1}}{n} \mp(n-1) \sqrt{\left(\frac{a_{1}}{n}\right)^{2}-\frac{2 a_{2}}{n(n-1)}} .
$$

The intersection by the straight line (22) on the $x$-axis is the point

$$
x_{a}=-\frac{a_{1}}{n} \pm(n-1) \sqrt{\left(\frac{a_{1}}{n}\right)^{2}-\frac{2 a_{2}}{n(n-1)}} .
$$

We have two points on $x$-axis:

$$
x_{a}^{-}=-\frac{a_{1}}{n}-(n-1) \sqrt{\left(\frac{a_{1}}{n}\right)^{2}-\frac{2 a_{2}}{n(n-1)}}
$$

and

$$
x_{a}^{+}=-\frac{a_{1}}{n}+(n-1) \sqrt{\left(\frac{a_{1}}{n}\right)^{2}-\frac{2 a_{2}}{n(n-1)}} .
$$

If all zeros of polynomial (13) are real, it is known that they lie on $x$-axis between $x_{a}^{-}$and $x_{a}^{+}$.

The tangent of the curve (14) at the point $x_{k}$ for large $\left|x_{k}\right|$ intersects with the $x$-axis at the point

$$
x_{t}=-\frac{a_{1}}{n} \pm(n-1) \sqrt{\left(\frac{a_{1}}{n}\right)^{2}-\frac{2 a_{2}}{n(n-1)}}+O\left(\frac{1}{x_{k}}\right),
$$

where for $c$ we used the relation (19).

We can show the above result in the form:

If the initial approximative value for zero $r$ of the polynomial (13) takes value $x_{k}$ for large $\left|x_{k}\right|$, then in the first following step of Laguerre formula (15), respectively (16), we obtain the value

$$
\begin{aligned}
x_{k+1} & =x_{t}=-\frac{a_{1}}{n} \pm(n-1) \sqrt{\left(\frac{a_{1}}{n}\right)^{2}-\frac{2 a_{2}}{n(n-1)}}+O\left(\frac{1}{x_{k}}\right) \\
& \approx-\frac{a_{1}}{n} \pm(n-1) \sqrt{\left(\frac{a_{1}}{n}\right)^{2}-\frac{2 a_{2}}{n(n-1)}},
\end{aligned}
$$

that is the value which is relatively close to the value of its largest, also the smallest zero of polynomial (13).

This way, we give an explanation of known characteristics of Laguerre formula (15), respectively (16).

It is known that the Laguerre method converges globally and monotonically to roots of polynomials if all the roots are real and different.

The Laguerre method (16) is obtained also in [3] as a particular case of a family of iterative formulas. 


\section{REFERENCES}

[1] E.N. Laguerre, Oeuvres de Laguerre, Vol. 1, Paris 1898, 87-103.

[2] E.Hansen, M. Patrick, A family of root finding methods, Numer. Math. 27(1977), 257-269.

[3] D.M. Simeunović, A procedure for obtaining a family of iterative formulas of higher order, Math. Moravica 13-2(2009), 23-26.

Dragomir M. Simeunović Mike Alasa 8

11000 BELGRADE

Serbia 